\title{
SLC25A38 Congenital Sideroblastic Anemia: Phenotypes and genotypes of 31 individuals from 24 families, including 11 novel mutations, and a review of the literature
}

Matthew Heeney ${ }^{1}$, Simon Berhe ${ }^{2}$, Dean Campagna ${ }^{2}$, Joseph Oved $^{3}$, Peter Kurre ${ }^{3}$, Peter Shaw $^{4}$, Juliana Teo ${ }^{4}$, Mayada Abu Shanap ${ }^{5}$, Hoda Hassab ${ }^{6}$, Bertil Glader ${ }^{7}$, Sanjay Shah ${ }^{8}$, Ayami Yoshimi ${ }^{9}$, Afshin Ameri ${ }^{10}$, Joseph Antin ${ }^{11}$, Jeanne Boudreaux ${ }^{12}$, Michael Briones ${ }^{12}$, Kathryn Dickerson ${ }^{13}$, Conrad Fernandez ${ }^{14}$, Roula Farah ${ }^{15}$, Henrik Hasle ${ }^{16}$, Sioban Keel ${ }^{17}$, Timothy Olson ${ }^{3}$, Jacquelyn Powers ${ }^{18}$, Melissa Rose ${ }^{19}$, Akiko Schimamura ${ }^{1}$, Sylvia Bottomley $^{20}$, and Mark Fleming ${ }^{21}$

${ }^{1}$ Dana-Farber/Boston Children's Cancer and Blood Disorders Center

${ }^{2}$ Boston Children's Hospital Department of Pathology

${ }^{3}$ The Children's Hospital of Philadelphia

${ }^{4}$ Children's Hospital at Westmead

${ }^{5}$ King Hussein Medical Center

${ }^{6}$ Alexandria University

${ }^{7}$ Lucile Packard Children's Hospital at Stanford Pediatrics

${ }^{8}$ Phoenix Children's Hospital Center for Cancer and Blood Disorders

${ }^{9}$ University of Freiburg

${ }^{10}$ Augusta University

${ }^{11}$ Dana-Farber Cancer Institute

${ }^{12}$ Children's Healthcare of Atlanta Inc

${ }^{13}$ The University of Texas Southwestern Medical Center

${ }^{14}$ Dalhousie University

${ }^{15}$ Lebanese American University

${ }^{16}$ Aarhus University Hospital

${ }^{17}$ Seattle Cancer Care Alliance

${ }^{18}$ Texas Children's Hospital

${ }^{19}$ Nationwide Children's Hospital

${ }^{20}$ The University of Oklahoma College of Medicine

${ }^{21}$ Boston Children's Hospital

February 4, 2021

\footnotetext{
Abstract

The congenital sideroblastic anemias (CSAs) are a heterogeneous group of inherited disorders of erythropoiesis characterized by pathologic deposits of iron in the mitochondria of developing erythroblasts. Mutations in the mitochondrial glycine carrier SLC25A38 cause the most common recessive form of CSA. Nonetheless, the disease is still rare, there being fewer than 70 reported families. Here we describe the clinical phenotype and genotypes of 31 individuals from 24 families, including 11 novel
} 
mutations. We also review the spectrum of reported mutations and genotypes associated with the disease, describe the unique localization of missense mutations in transmembrane domains and account for the reoccurrence of several alleles in different populations.

\section{MUTATION UPDATE SLC25A38}

SLC25A38 Congenital Sideroblastic Anemia : Phenotypes and genotypes of 31 individuals from 24 families, including 11 novel mutations, and a review of the literature

Matthew M. Heeney ${ }^{1^{*}}$, Simon Berhe ${ }^{2 *}$, Dean R. Campagna ${ }^{2 *}$, Joseph H. Oved ${ }^{3}$, Peter Kurre ${ }^{4}$, Peter J. Shaw ${ }^{5}$, Juliana Teo ${ }^{6}$, Mayada Abu Shanap ${ }^{7}$, Hoda M. Hassab ${ }^{8}$, Bertil E. Glader ${ }^{9}$, Sanjay Shah ${ }^{10}$, Ayami Yoshimi ${ }^{11}$, Afshin Ameri ${ }^{12}$, Joseph H. Antin ${ }^{13}$, Jeanne Boudreaux ${ }^{14}$, Michael Briones ${ }^{14}$, Kathryn E. Dickerson ${ }^{15}$, Conrad V. Fernandez ${ }^{16}$, Roula Farah ${ }^{17}$, Henrik Hasle ${ }^{18}$, Sioban B. Keel ${ }^{19}$, Timothy S. Olson $^{20}$, Jacquelyn M. Powers ${ }^{21}$, Melissa J. Rose ${ }^{22}$, Akiko Shimamura ${ }^{1}$, Sylvia S. Bottomley ${ }^{23}$, Mark D. Fleming ${ }^{2}$

${ }^{1}$ Division of Hematology, Dana-Farber Boston Children's Cancer and Blood Disorders Center and Department of Pediatrics, Harvard Medical School, Boston, MA, USA

${ }^{2}$ Department of Pathology, Boston Children's Hospital and Harvard Medical School, Boston, MA, USA

${ }^{3}$ Cellular Therapy and Transplant Section, Division of Oncology and Comprehensive Bone Marrow Failure Center, Division of Hematology, Children's Hospital of Philadelphia, Philadelphia, PA, USA

${ }^{4}$ Pediatric Comprehensive Bone Marrow Failure Center, Children's Hospital of Philadelphia, Philadelphia, PA, USA

${ }^{5}$ BMT Services, Children's Hospital at Westmead; Faculty of Medicine and Health, University of Sydney, Sydney, AU

${ }^{6}$ Department of Haematology, Children's Hospital at Westmead, Sydney, AU

${ }^{7}$ King Hussein Medical Center, Amman, Jordan

${ }^{8}$ Department of Paediatrics, Faculty of Medicine, Alexandria University, Alexandria, Egypt

${ }^{9}$ Division of Hematology-Oncology, Lucille Packard Children's Hospital, Stanford, CA, USA

${ }^{10}$ Center for Cancer and Blood Disorders, Phoenix Children's Hospital, Phoenix, AZ, USA

${ }^{11}$ Department of Paediatrics and Adolescent Medicine, Division of Paediatric Haematology and Oncology, Medical Center, Faculty of Medicine, University of Freiburg, Freiburg, Germany

${ }^{12}$ Division of Pediatric Hematology/Oncology, Department of Pediatrics, Augusta University, Augusta, GA, USA

${ }^{13}$ Hematopoietic Stem Cell Transplantation Program, Dana-Farber Cancer Institute, and Harvard Medical School, Boston, MA, USA

${ }^{14}$ Aflac Cancer and Blood Disorders Center, Children's Healthcare of Atlanta and Emory University, Atlanta, GA, USA

${ }^{15}$ Department of Pediatrics, University of Texas Southwestern, Dallas, TX, USA

${ }^{16}$ Division of Hematology-Oncology, IWH Center, Dalhousie University, Halifax, NS, Canada

${ }^{17}$ Department of Pediatrics, Lebanese American University Medical Center, Beirut, Lebanon

${ }^{18}$ Department of Pediatrics, Aarhus University Hospital, Aarhus University, Aarhus, Denmark

${ }^{19}$ Division of Hematology, Department of Medicine, University of Washington and Seattle Cancer Care Alliance, Seattle, WA, USA 
${ }^{20}$ Cellular Therapy and Transplant Section, Division of Oncology and Comprehensive Bone Marrow Failure Center, Division of Hematology, Children's Hospital of Philadelphia, Philadelphia, PA, USA

${ }^{21}$ Texas Children's Hospital and Department of Pediatrics, Section of Hematology/Oncology, Baylor College of Medicine, Houston, TX, USA

${ }^{22}$ Division of Hematology \& Oncology, Nationwide Children's Hospital, Department of Pediatrics, The Ohio State University, Columbus, OH, USA

${ }^{23}$ Hematology-Oncology Section, University of Oklahoma College of Medicine, Oklahoma City, OK, USA

*These authors contributed equally.

Funding sources: R01 DK087992 and American Society of Hematology Bridge Grant (M.D.F) and RC2 DK122533 (M.D.F. and A.S.)

\begin{abstract}
The congenital sideroblastic anemias (CSAs) are a heterogeneous group of inherited disorders of erythropoiesis characterized by pathologic deposits of iron in the mitochondria of developing erythroblasts. Mutations in the mitochondrial glycine carrier SLC25A38 cause the most common recessive form of CSA. Nonetheless, the disease is still rare, there being fewer than 70 reported families. Here we describe the clinical phenotype and genotypes of 31 individuals from 24 families, including 11 novel mutations. We also review the spectrum of reported mutations and genotypes associated with the disease, describe the unique localization of missense mutations in transmembrane domains and account for the presence of several alleles in different populations.
\end{abstract}

\title{
KEY WORDS:
}

Sideroblastic anemia, Genetics, Iron, Erythropoiesis, Hematopoietic Stem Cell Transplantation

\section{INTRODUCTION}

The congenital sideroblastic anemias (CSAs) are a heterogeneous group of inherited disorders of erythropoiesis characterized by pathologic deposits of iron in the mitochondria of developing erythroblasts (Cartwright \& Deiss, 1975). The genetically defined CSAs can be attributed to defects in three interrelated mitochondrial pathways: heme biosynthesis, iron-sulfur cluster assembly, and mitochondrial protein synthesis and respiration (Ducamp \& Fleming, 2019). CSAs due to primary heme biosynthesis defects are the most prevalent. The most common CSA, X-linked sideroblastic anemia (XLSA), is caused by mutations in the first and rate-limiting enzyme in erythroid heme synthesis, 5-aminolevulinate synthase 2 (ALAS2), which catalyses the condensation of glycine with succinyl coenzyme A to form 5-aminolevulinic acid (ALA) in the mitochondrial matrix. More than 200 families with XLSA have been described in the literature (Bottomley \& Fleming, 2014). ALAS2 is extraordinarily dependent upon high levels of glycine to ensure sufficient heme synthesis, as it has a very high $K_{m}(9.3 \pm 1.2 \mathrm{mM})$ for this substrate (Bishop, Tchaikovskii, Nazarenko, \& Desnick, 2013).

An autosomal recessive form due to loss-of-function mutations in SLC25A38 is the second most common form of CSA (Guernsey et al., 2009). SLC25A38 is a member of the Mitochondrial Solute Carrier Family 25 (SLC25) family of transporters (Kunji, 2004; Ruprecht \& Kunji, 2020), is encoded on chromosome 3 p22, and is highly and selectively expressed in erythroblasts (Guernsey et al., 2009). The yeast ortholog of SLC25A38 (yDL119c) is essential for efficient heme biosynthesis and knockdown of the orthologous proteins in zebrafish results in anemia; each of these phenotypes can be rescued by the addition of glycine or ALA (Fernandez-Murray et al., 2016; Guernsey et al., 2009). Transport studies show that yDL119c is a high affinity mitochondrial glycine importer (Lunetti et al., 2016).

While the severity of the anemia is generally far more profound than XLSA, consistent with the shared interruption of heme synthesis, the morphologic features of the blood and bone marrow in the SLC25A38 anemia are highly reminiscent of XLSA and characterized by a reticulocytopenic, hypochromic, microcytic 
anemia with a very wide red blood cell distribution width (RDW) and ring sideroblasts (RS) predominantly found in later erythroid precursors. To date, 69 families and a total of 36 different causative SLC25A38 mutations have been described (W. An et al., 2015; W. B. An et al., 2019; Andolfo et al., 2020; Fouquet et al., 2019; Guernsey et al., 2009; Kakourou et al., 2016; Kannengiesser et al., 2011; Kim, Shah, Bottomley, \& Shah, 2018; Kucerova et al., 2011; Le Rouzic et al., 2017; Liu et al., 2013; Mehri et al., 2018; Ravindra et al., 2020; Shefer Averbuch et al., 2018; Ulirsch et al., 2019; Uminski et al., 2020; Wong et al., 2015). Here we describe the clinical phenotypes and genotypes of an additional 31 individuals from 24 families, including 11 novel mutations. We also review the spectrum of mutations and genotypes associated with the disease, including describing the unique localization of missense (MS) mutations in transmembrane (TM) domains and account for the reoccurrence of several alleles in different populations.

\section{METHODS}

Patients with a diagnosis of CSA or an aregenerative congenital anemia were referred for research testing to M.D.F., M.M.H, or S.S.B., as a part of human subjects research protocols approved at Boston Children's Hospital and the University of Oklahoma. All subjects or their guardians provided written informed consent to participate in the study. The patients described here are a subset of 251 CSA probands referred to M.D.F., M.M.H., and S.S.B., which, in addition to the 24 families described here, includes 12 families described in the initial report of the SLC25A38 anemia. Most mutations were discovered by research Sanger sequencing of SLC25A38 exons. Several were identified by commercial inherited anemia NextGen sequencing panels. In most cases, mutations were proven to be biallelic based on sequencing of parental and/or sibling DNA samples.

Mutational and splicing analysis was performed with Alamut Visual (Sophia Genetics). Protein alignment and conservation analysis was performed using the Clustal Omega (https://www.ebi.ac.uk/Tools/msa/clustalo/) set of multiple sequence alignment tools and the sequences described in Supplementary Tables 1 and 2. Statistical analysis was performed with Prism 9 for macOS (GraphPad Software, LLC).

SLC25A38 CSA patients described in the literature accessible through PubMed and available online are reviewed and are current as of January 25, 2021.

\section{RESULTS}

\section{Clinical Features}

We identified 31 patients (16 males and 15 females) from 24 families with proven or presumptive biallelic SLC25A38 rare variants predicted to be functionally deleterious (Tables 1 and 2). Using Sanger or whole exome sequencing in 70 additional CSA probands without a specific genetic diagnosis, we did not identify an individual with even a single $S L C 25 A 38$ allele present in reference databases at a frequency of $<0.1$, nor did we find evidence of a deletion of all or part of the gene. This suggests that pathogenic SLC25A38 alleles that are occult to sequencing exons are unusual.

Two patients (13.1 and 22.4) presented with hydrops fetalis in utero. Sixteen presented in the neonatal period ( $<1$ week of life), 10 in infancy ( $<12$ months), and one each at 4- (4.1) and 14-years of age (14.1); the age of clinical presentation of one patient is unknown. In 10 of 23 families where the family history was known, there was defined consanguinity. Five (5) of the remaining 13 families were not known to be consanguineous but originated from genetically isolated populations or populations with known founder effects (e.g., Acadians from the Canadian Maritime Provinces).

All patients presented with reticulocytopenic, microcytic anemia (Table 1). In the 13 individuals in whom pre-transfusion $\mathrm{CBC}$ data are available, most of whom were neonates or infants at the time, the mean hemoglobin (HGB) was $5.1 \pm 2.4 \mathrm{~g} / \mathrm{dL}$ (generalized normal range 10.5-13.0 g/dL), mean cell volume (MCV) $61.9 \pm 4.9 \mathrm{fL}$ (generalized normal range 79.6-83.3 fL), and absolute reticulocyte count $0.032 \pm 0.032 \mathrm{x}$ $10^{6} / \mu \mathrm{L}$ (generalize normal range $0.037-0.104 \times 10^{6} / \mu \mathrm{L}$ ). In 20 of 22 families the diagnosis of CSA was made in the proband by bone marrow aspiration where ring sideroblasts generally constituted $>15 \%$ of nucleated 
erythroblasts. In several cases, RS were absent or only rare. In most, but not all, cases, there was an erythroid hyperplasia in the bone marrow; in some there was an erythroid hypoplasia. Conspicuous dyserythropoiesis and variable fibrosis were present in a minority of samples. Anemic siblings were sometimes diagnosed with CSA by genetic testing alone, as was the case with patient 20.2, whose older sibling (20.1), has previously been reported (Kim et al., 2018). Three patients from two families (patients 2.1, 2.2, and 18.1) were initially regarded as having an atypical form of Diamond-Blackfan anemia (DBA). In family 2, the eventual identification of rare siderocytes in the peripheral blood suggested CSA, which was confirmed by candidate gene sequencing. Because of the unusual, apparently syndromic features in patient 14.1, this patient's diagnosis was established by whole exome sequencing. In patient 18.1, SLC25A38 mutations were identified by whole exome sequencing years after successful hematopoietic stem cell transplantation for "DBA." Most patients did not have abnormalities in other organ systems that were not attributable to chronic anemia or iron overload (e.g. growth failure, endocrine abnormalities, liver disease, cardiomyopathy), but several potentially syndromic features were observed in a number of patients (Table 1): unilateral corneal clouding (13.1), a "box-shaped" hyperostotic skull $(14.1,24.1)$, macrocephaly $(8.2,17.1)$, syndromic facies (28.1), meningomyelocoele/club foot (17.1), genital abnormalities (18.1, 22.3, 22.4), behavioral issues (18.1 and $22.1)$, aortic root/coronary abnormalities $(20.2,22.2,22.3)$.

\section{Therapy}

All of the patients have required transfusions, most chronically and beginning in the neonatal period or infancy (Table 3). Of the 28 patients in whom data are available, 2 patients received their first transfusions in utero (13.1 and 22.4), 12 in the neonatal period, 11 in infancy, and 3 between age 4- and 8-years. All but one was maintained on regular transfusions with a transfusion interval of between 2 and 8 weeks. In 20 of 20 patients for whom oral pyridoxine was prescribed, there was no improvement in the hemoglobin (HGB). All patients surviving early childhood have developed secondary iron overload and have required chelation. A variety of agents including deferoxamine, deferisirox, deferiprone alone or in combination have been employed. One patient (2.1), poorly compliant with chelation, died at age 18 from cardiomyopathy. Another (17.1), age 3, died of line-associated sepsis. Three patients who were status-post splenectomy experienced thrombocytosis and/or thrombotic events. The median age of patients alive at the time of last follow-up is 11 years (range 1-39 years).

Nine patients have undergone allogeneic hematopoietic stem cell transplantation (Table 4) with a median follow up of 7 years (range 1 mos. to $17 \mathrm{yrs}$.). All transplanted patients are alive; 8 of 9 had full engraftment and became transfusion independent. One patient (14.1) had secondary graft failure at 18-months posttransplant with auto recovery; she is transfusion dependent and being prepared for second transplant. Four patients received myeloablative conditioning, and 4 other received reduced intensity conditioning. Donors were matched unrelated donor $(\mathrm{n}=4)$, matched related donors (one matched family donor and three were matched sibling donors), and one patient had one antigen mismatch sibling donor. Methotrexate and a calcineurin inhibitors were the most common graft versus host disease prophylaxis. Acute and chronic graft versus host disease were seen in 1 and 3 patients, respectively. One patient developed chronic post-transplant autoimmune hemolytic anemia requiring transfusion.

Mutation Analysis

The 24 families carry 27 distinct SLC25A38 mutations (Table 2). Sixteen (16) of these mutations have been described by us and others previously. Eleven (11) mutations are novel, including one MS allele (c.388G >A; p.Gly130Arg), 5 frameshift (FS) alleles (c.207_214del, p.Met70Cysfs*80; c.362del, p.Pro121Glnfs*26; c.475del, p.Glu159Argfs*7; c.669_682del, p.Cys223Trpfs*67; and c.809dup, p.Phe271Leufs*24), and 5 variants predicted to interrupt splicing (c.70-2A $>$ C, c. $276+1 \mathrm{G}>\mathrm{A}$, c.277-2A $>$ C, c. $457-1 \mathrm{G}>\mathrm{T}$, and c. $792+5 \mathrm{G}>\mathrm{C}$ ). In contrast to many of the previously reported pathogenic alleles, which are generally more common (see below), only one of these variants occurs in a sequence with a predisposition to mutation: the c.207_214del involves a deletion of a 7 base-pair direct repeat. Furthermore, only two of the novel mutations, c.457-1G $>$ T (rs1448237170, MAF 4.00x10-6) and c.669_682del (rs781372292, MAF $1.77 \times 10^{-5}$ ), are recorded in references databases such as gnomAD (gnomad.broadinstitute.org). 
As expected in a rare recessive disease, 19 of the 24 families (79\%) are homozygous for the pathogenic mutation. In the patients with homozygous mutations, 10 are known to be consanguineous and 5 are from geographically or ethnically restricted populations that may be genetically less diverse.

In this cohort, we detected no difference in age of onset of anemia, age at initial transfusion, pre-transfusion HGB, or transfusion interval among patients with two null alleles, two splicing alleles, or at least one MS mutation (data not shown).

The SLC25A38 mutation spectrum

Currently, there are 16 publications, including the current one, describing, a total of 92 SLC25A38 CSA families from diverse geographic and ethnic backgrounds (Table 5). As is true of our sample, approximately three-quarters (77\%) of the reported probands carry homozygous mutant alleles (Figure 1A). In one case, homozygosity is the result of constitutional uniparental isodisomy (Andolfo et al., 2020). MS (36\%), frameshift $(27 \%)$ and stop-gained $(27 \%)$ alleles each constitute one-quarter to one-third of alleles detected in probands (Figure 1B). Variants predicted to affect splicing (9\%) or cause a stop-loss (EXT, 1\%) are comparatively rare. Two MS variants, c.560G $>$ C; p.Arg187Pro and c.625G $>$ C; p.Asp209His, are also predicted to affect splicing, the former likely activating a cryptic splice acceptor site within exon 5 and the latter altering the conserved $\mathrm{G}$ at the last base pair of exon 5. Patients homozygous for MS mutations are most common, constituting approximately one-third (31\%) of all reported probands (Figure 1C). Whereas $42 \%$ of patients bear at least one MS allele and may retain some transport function, $46 \%$ have two stop-gained or frameshift (or a combination of both) presumptive null alleles, and another $12 \%$ have two splicing alleles or a splicing allele in trans of a frameshift or stop-loss allele, also likely to retain little transport activity (Figure 1D).

Of the 47 reported disease-associated mutations, 12 occur at sequences prone to recurrence, including 9 at $\mathrm{CpG}$ dinucleotides and 3 at a direct or simple repeat. Of the 21 apparently recurrent mutations, 9 are at a CpG or repeat (Figure 2).

Pathogenic MS mutations are distributed nearly exclusively in the transmembrane (TM) domains. Of the 21 pathogenic MS mutations, 19 are located in amino acids within a TM domain (Table 5 and Figure 2). One of the remaining two, c.625G $>$ C; p.Asp209His, is also predicted to affect splicing. The remaining variant, c.469G >C; p.Gly157Arg, in addition to be located between TM3 and TM4, is conserved neither in SLC25 family members, nor in SLC25A38 orthologues. There is no difference in the relative conservation of amino acids in TM and non-TM regions of SLC25A38 orthologues (Mann-Whitney $\mathrm{P}=0.385$ ) whereas there is an unexpected predominance of disease-causing mutations present in TMs $\left(\chi^{2} \mathrm{P}<0.001\right)$. Of the TM residues with pathogenic mutations, there is a trend toward being relatively conserved compared to other TM amino acids (Mann-Whitney $\mathrm{P}=0.085$ )

\section{DISCUSSION}

This is the largest series of patients with SLC25A38 associated CSA yet reported, describing 31 individuals from 24 different families and 11 novel mutations, expanding the total number of reported families and pathogenic alleles to 92 and 47 , respectively.

Despite the diversity of mutations, there are several unexpected aspects of the SLC25A38 anemia revealed by these studies worth noting. First is the very limited evidence that there is a genotype-phenotype correlation. Essentially all patients present at birth or infancy with a severe hypochromic, microcytic anemia that eventually requires chronic transfusion. This is in stark contrast with the most common form of hypochromic microcytic, non-syndromic CSA, XLSA, which is the major differential diagnosis. Male patients with XLSA may present at birth to older adulthood. The most severe XLSA cases tend to present at an earlier age, but it is unusual for a patient to have transfusion dependent anemia as is typical of SLC25A38 disease. Indeed, the anemia in XLSA is frequently incidental and may be discovered only by screening or as a result of investigation of unexplained iron overload. There are, however, several exceptional cases of patients with SLC25A38 disease coming to medical attention in their teens or twenties. Three of these patients had homozygous mutations at codon 134 [p.Arg134His or p.Arg134Cys] (Fouquet et al., 2019; Hanina, Bain, Clark, \& Layton, 
2018; Le Rouzic et al., 2017). However, two other patients homozygous for the p.Arg134Cys allele presented at age 2 months and 2 years (W. An et al., 2015; W. B. An et al., 2019; Kannengiesser et al., 2011). In our own cohort, a patient with a homozygous variant at the only incompletely conserved +5 position of a splice donor site $($ c. $792+5 \mathrm{G}>\mathrm{C})$ presented in his mid-teens. It is certainly possible that other genotype-phenotype correlations are masked by the clinical imperative to initiate transfusions in a patient with a HGB less than $\sim 9 \mathrm{~g} / \mathrm{dL}$. Indeed, in the publications in which an initial diagnostic HGB is reported, only one individual, a neonate, had a hemoglobin >9 g/dL (Guernsey et al., 2009; Hanina et al., 2018; Kannengiesser et al., 2011; Liu et al., 2013; Wong et al., 2015).

Although clinical practice to transfuse these patients may disguise the subtle differences between SLC25A38 genotypes, it is abundantly evident that an SLC25A38 null genotype does not preclude some mitochondrial glycine being available for heme synthesis. This may be due to other, less specific transporters, possibly including the highly homologous protein SLC25A39, or pathways that produce glycine from other amino acids, such as serine (Amelio, Cutruzzola, Antonov, Agostini, \& Melino, 2014; Kory et al., 2018). Leveraging these pathways may provide an avenue for therapy. However, based on limited data, it is unlikely that glycine supplementation alone will suffice (Fernandez-Murray et al., 2016; LeBlanc et al., 2016).

The SLC25A38 anemia is regarded as non-syndromic. Nevertheless, twelve of the 31 patients described here had developmental or intellectual disabilities. Similar abnormalities, including psychomotor delay, hypotonia, facial dysmorphism (Fouquet et al., 2019), Hypospadias (W. An et al., 2015), congenital myelomeningocele, patent ductus arteriosus and ventricular septal defects (Wong et al., 2015) have been reported in several other patients, but no abnormality is unusually prevalent across multiple families to suggest a specific syndromic association.

Three patients who underwent splenectomy developed thrombocytosis and/or recurrent thrombosis, further supporting the notion that splenectomy may be contraindicated in SLC25A38 CSA as has generally been advocated in other patients with microcytic CSAs (Bottomley \& Fleming, 2014; Fouquet et al., 2019).

The 47 described SLC25A38 pathogenic mutations occur at 40 different codons. However, nearly one-third (27 of 92) families carry at least one copy of either the c.324_325del or the c.349C>T allele. The latter has been identified in families of Acadian (Guernsey et al., 2009), African American (current report), South Asian (Ravindra et al., 2020), Greek (Guernsey et al., 2009), and Northern European (this report) origin, suggesting that it has reoccurred on multiple occasions. In fact, in one case (21.1), the patient is homozygous for the c.349C $>\mathrm{T}$ allele, associated null allele, but one copy also carries a MS variant in a non-conserved residue in cis (c.[161G > A;349C > T]; p. [Arg54His;Arg117X]). Reanalysis of other SLC25A38 anemia patients previously reported by us (Guernsey et al., 2009) identified one other patient having this compound mutant chromosome (data not shown). Both of these variants occur at hypermutable cytosine-guanosine (CpG) dinucleotides. This would support the notion that the p.Arg117X allele has occurred on multiple occasions and that homozygosity for a disease-associated variant in SLC25A38 should not necessarily be taken as evidence of identity by descent.

Because of the severity and great similarity of the disorder to transfusion-dependent $\beta$-thalassemia (thalassemia major), all of the patients in our cohort were at one time managed with transfusion and iron chelation in a manner similar to thalassemia. This is similarly true of nearly all of the patients described in the literature. Just as we identified no consistent, distinctive syndromic aspects of the disease outside the anemia, we did not observe any complications or undue toxicity as a result of transfusion or iron overload. The oldest patient in this cohort was 39 years of age, and we are aware of at least two patients in their early fifties (Guernsey et al., 2009), suggesting that modern transfusion and chelation regimens support long-term survival.

Nonetheless, 9 of our patients underwent allogenic HSCT at varying times during their disease course. Ten other patients (Guernsey et al., 2009; Kannengiesser et al., 2011; Uminski et al., 2020) as well as the sibling of one patient included in this series (Kim et al., 2018) are reported to have been transplanted. Comprehensive details are unavailable for most of these patients, but when stated, similar to those described here, HSCT 
regimens have variously included matched-related and matched unrelated donors with fully myeloablative or reduced intensity conditioning regimens. In many cases, aggressive chelation was employed to reduce the iron burden pre-transplant and phlebotomy was often used post-HSCT to further normalize iron stores. In all, of 18 patients receiving HSCT, 14 have achieved transfusion independence, 3 grafts failed, and one patient died in the immediate post-transplant period with follow up ranging from months to 19 years. In no case has an unusual disease-specific transplant-related morbidity been reported. Thus, allogeneic HSCT provides a curative option in SLC25A38 CSA, and may be considered in young patients with appropriate donors prior to the development of sequelae of chronic transfusions such as hemosiderosis and alloimmunization.

\section{CONCLUSION}

Although it is uncommon, SLC25A38 CSA is a clinically distinctive entity nearly always associated with transfusion-dependent microcytic, hypochromic sideroblastic anemia from infancy. Although it is presently managed in a manner similar to other severe anemias, such as $\beta$-thalassemia major, with chronic transfusion and iron chelation, HSCT may be an increasingly attractive option for definitive therapy. Furthermore, as disease-specific metabolic and genetic therapies for rare diseases emerge, SLC25A38 CSA will be increasingly important to distinguish genetically.

\section{ACKNOWLEDGMENTS}

The authors thank the patients and their families for participating. Susan Wong is acknowledged for administration of human subjects research protocols.

\section{CONFLICT OF INTEREST STATEMENT}

The authors declare no competing interests

\section{DATA AVAILABILITY}

All primary sequence data are available upon request.

\section{REFERENCES}

Amelio, I., Cutruzzola, F., Antonov, A., Agostini, M., \& Melino, G. (2014). Serine and glycine metabolism in cancer. Trends Biochem Sci, 39 (4), 191-198. doi:10.1016/j.tibs.2014.02.004

An, W., Zhang, J., Chang, L., Zhang, Y., Wan, Y., Ren, Y., . . . Guo, Y. (2015). Mutation analysis of Chinese sporadic congenital sideroblastic anemia by targeted capture sequencing.J Hematol Oncol, 8 , 55. doi:10.1186/s13045-015-0154-0

An, W. B., An, W. B., Liu, C., Wan, Y., Guo, Y., Wang, S. C., . . . Zhu, X. F. (2019). [Clinical features and gene mutation spectrum in children with sideroblastic anemia].Zhongguo Dang Dai Er Ke Za Zhi, 21 (10), 1016-1021.

Andolfo, I., Martone, S., Ribersani, M., Bianchi, S., Manna, F., Genesio, R., . . . Russo, R. (2020). Apparent recessive inheritance of sideroblastic anemia type 2 due to uniparental isodisomy at the SLC25A38 locus. Haematologica . doi:10.3324/haematol.2020.258533

Bishop, D. F., Tchaikovskii, V., Nazarenko, I., \& Desnick, R. J. (2013). Molecular expression and characterization of erythroid-specific 5-aminolevulinate synthase gain-of-function mutations causing X-linked protoporphyria. Mol Med, 19 , 18-25. doi:10.2119/molmed.2013.00003

Bottomley, S. S., \& Fleming, M. D. (2014). Sideroblastic anemia: diagnosis and management. Hematol Oncol Clin North Am, 28 (4), 653-670, v. doi:10.1016/j.hoc.2014.04.008

Cartwright, G. E., \& Deiss, A. (1975). Sideroblasts, siderocytes, and sideroblastic anemia. N Engl J Med, 292 (4), 185-193. doi:10.1056/nejm197501232920405

Ducamp, S., \& Fleming, M. D. (2019). The molecular genetics of sideroblastic anemia. Blood, 133 (1), 59-69. doi:10.1182/blood-2018-08-815951 
Fernandez-Murray, J. P., Prykhozhij, S. V., Dufay, J. N., Steele, S. L., Gaston, D., Nasrallah, G. K., . . . McMaster, C. R. (2016). Glycine and Folate Ameliorate Models of Congenital Sideroblastic Anemia. PLoS Genet, 12 (1), e1005783. doi:10.1371/journal.pgen.1005783

Fouquet, C., Le Rouzic, M. A., Leblanc, T., Fouyssac, F., Leverger, G., Hessissen, L., . . . Ducassou, S. (2019). Genotype/phenotype correlations of childhood-onset congenital sideroblastic anaemia in a European cohort. Br J Haematol, 187 (4), 530-542. doi:10.1111/bjh.16100

Guernsey, D. L., Jiang, H., Campagna, D. R., Evans, S. C., Ferguson, M., Kellogg, M. D., . . . Samuels, M. E. (2009). Mutations in mitochondrial carrier family gene SLC25A38 cause nonsyndromic autosomal recessive congenital sideroblastic anemia.Nat Genet, 41 (6), 651-653. doi:10.1038/ng.359

Hanina, S., Bain, B. J., Clark, B., \& Layton, D. M. (2018). Congenital sideroblastic anemia in a female.Am J Hematol, 93 (9), 1181-1182. doi:10.1002/ajh.25196

Kakourou, G., Vrettou, C., Kattamis, A., Destouni, A., Poulou, M., Moutafi, M., . . . Traeger-Synodinos, J. (2016). Complex preimplantation genetic diagnosis for beta-thalassaemia, sideroblastic anaemia, and human leukocyte antigen (HLA)-typing. Syst Biol Reprod Med, 62 (1), 69-76. doi:10.3109/19396368.2015.1100692

Kannengiesser, C., Sanchez, M., Sweeney, M., Hetet, G., Kerr, B., Moran, E., . . . May, A. (2011). Missense SLC25A38 variations play an important role in autosomal recessive inherited sideroblastic anemia. Haematologica, 96 (6), 808-813. doi:10.3324/haematol.2010.039164

Kim, M. H., Shah, S., Bottomley, S. S., \& Shah, N. C. (2018). Reduced-toxicity allogeneic hematopoietic stem cell transplantation in congenital sideroblastic anemia. Clin Case Rep, 6 (9), 1841-1844. doi:10.1002/ccr3.1667

Kory, N., Wyant, G. A., Prakash, G., Uit de Bos, J., Bottanelli, F., Pacold, M. E., . . . Sabatini, D. M. (2018). SFXN1 is a mitochondrial serine transporter required for one-carbon metabolism. Science, 362 (6416). doi:10.1126/science.aat9528

Kucerova, J., Horvathova, M., Mojzikova, R., Belohlavkova, P., Cermak, J., \& Divoky, V. (2011). New mutation in erythroid-specific delta-aminolevulinate synthase as the cause of X-linked sideroblastic anemia responsive to pyridoxine.Acta Haematol, 125 (4), 193-197. doi:10.1159/000322870

Kunji, E. R. (2004). The role and structure of mitochondrial carriers. FEBS Lett, 564 (3), 239-244. doi:10.1016/S0014-5793(04)00242-X

Le Rouzic, M. A., Fouquet, C., Leblanc, T., Touati, M., Fouyssac, F., Vermylen, C., . . . Vannier, J. P. (2017). Non syndromic childhood onset congenital sideroblastic anemia: A report of 13 patients identified with an ALAS2 or SLC25A38 mutation. Blood Cells Mol Dis, 66 , 11-18. doi:10.1016/j.bcmd.2017.07.003

LeBlanc, M. A., Bettle, A., Berman, J. N., Price, V. E., Pambrun, C., Yu, Z., . . . Fernandez, C. V. (2016). Study of Glycine and Folic Acid Supplementation to Ameliorate Transfusion Dependence in Congenital SLC25A38 Mutated Sideroblastic Anemia. Pediatr Blood Cancer, 63 (7), 1307-1309. doi:10.1002/pbc.25981

Liu, G., Guo, S., Kang, H., Zhang, F., Hu, Y., Wang, L., . . . Nie, G. (2013). Mutation spectrum in Chinese patients affected by congenital sideroblastic anemia and a search for a genotype-phenotype relationship. Haematologica, 98 (12), e158-160. doi:10.3324/haematol.2013.095513

Lunetti, P., Damiano, F., De Benedetto, G., Siculella, L., Pennetta, A., Muto, L., . . . Capobianco, L. (2016). Characterization of Human and Yeast Mitochondrial Glycine Carriers with Implications for Heme Biosynthesis and Anemia. J Biol Chem, 291 (38), 19746-19759. doi:10.1074/jbc.M116.736876

Mehri, M., Zarin, M., Ardalani, F., Najmabadi, H., Azarkeivan, A., \& Neishabury, M. (2018). Novel mutations in mitochondrial carrier family gene SLC25A38, causing congenital sideroblastic anemia in Iranian families, identified by whole exome sequencing. Blood Cells Mol Dis, 71 , 39-44. doi:10.1016/j.bcmd.2018.02.002 
Ravindra, N., Athiyarath, R., S, E., S, S., Kulkarni, U., N, A. F., . . . Edison, E. S. (2020). Novel frameshift variant (c.409dupG) in SLC25A38 is a common cause of congenital sideroblastic anaemia in the Indian subcontinent. J Clin Pathol . doi:10.1136/jclinpath-2020-206647

Ruprecht, J. J., \& Kunji, E. R. S. (2020). The SLC25 Mitochondrial Carrier Family: Structure and Mechanism. Trends Biochem Sci, 45 (3), 244-258. doi:10.1016/j.tibs.2019.11.001

Shefer Averbuch, N., Steinberg-Shemer, O., Dgany, O., Krasnov, T., Noy-Lotan, S., Yacobovich, J., . . . Tamary, H. (2018). Targeted next generation sequencing for the diagnosis of patients with rare congenital anemias. Eur J Haematol, 101 (3), 297-304. doi:10.1111/ejh.13097

Ulirsch, J. C., Verboon, J. M., Kazerounian, S., Guo, M. H., Yuan, D., Ludwig, L. S., . . . Gazda, H. T. (2019). The Genetic Landscape of Diamond-Blackfan Anemia. Am J Hum Genet, 104 (2), 356. doi:10.1016/j.ajhg.2018.12.011

Uminski, K., Houston, D. S., Hartley, J. N., Liu, J., Cuvelier, G. D. E., \& Israels, S. J. (2020). Clinical characterization and hematopoietic stem cell transplant outcomes for congenital sideroblastic anemia caused by a novel pathogenic variant in SLC25A38. Pediatr Blood Cancer, e28623. doi:10.1002/pbc.28623

Wong, W. S., Wong, H. F., Cheng, C. K., Chang, K. O., Chan, N. P., Ng, M. H., \& Wong, K. F. (2015). Congenital sideroblastic anaemia with a novel frameshift mutation in SLC25A38. J Clin Pathol, 68 (3), 249-251. doi:10.1136/jclinpath-2014-202211

\section{Hosted file}

SLC25A38 Mutation Update Figure Legends Final.pdf available at https://authorea.com/ users/393689/articles/507269-slc25a38-congenital-sideroblastic-anemia-phenotypes-andgenotypes-of-31-individuals-from-24-families-including-11-novel-mutations-and-a-reviewof-the-literature

A.

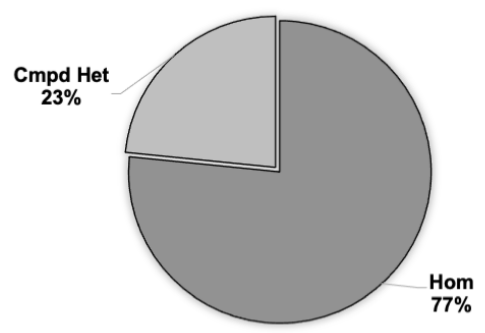

c.

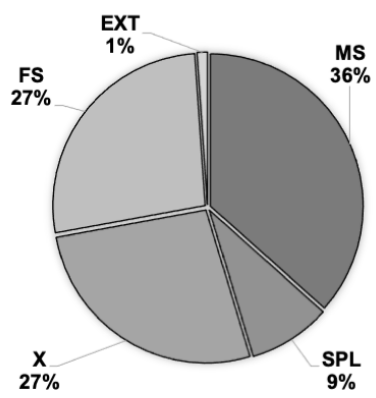

B.

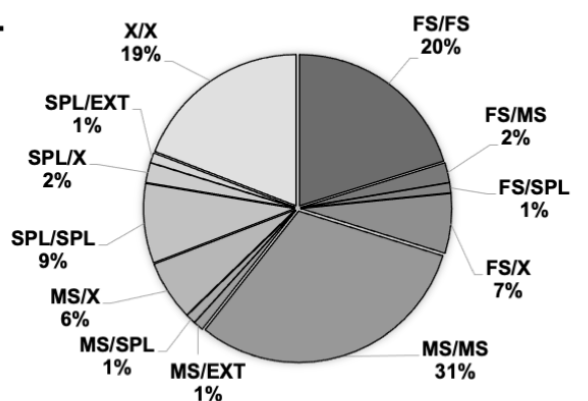

D.

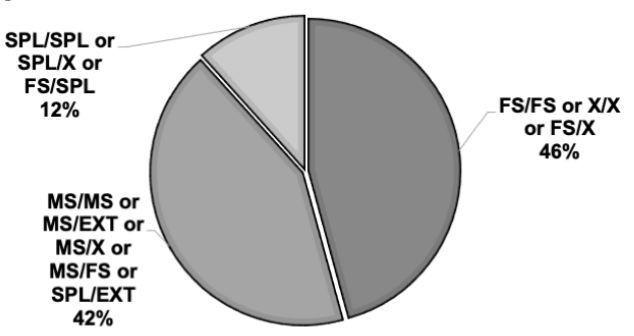




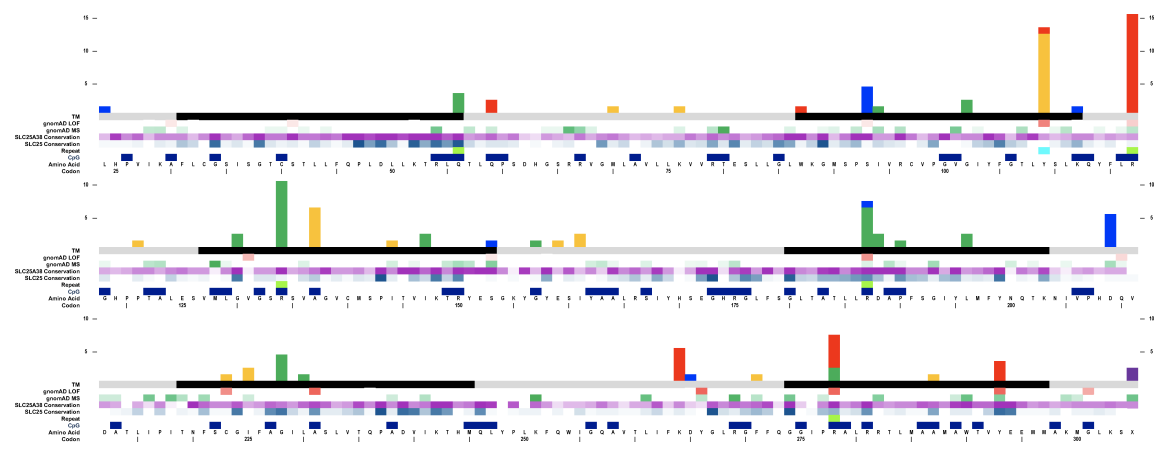

\section{Hosted file}

SLC25A38 Mutation Update Tables Plain Text Final.pdf available at https://authorea.com/ users/393689/articles/507269-slc25a38-congenital-sideroblastic-anemia-phenotypes-andgenotypes-of-31-individuals-from-24-families-including-11-novel-mutations-and-a-reviewof-the-literature 